\title{
Choice or Rank Data in Stated Preference Surveys?
}

\author{
Becky P.Y. Loo ${ }^{*}, 1$, S.C. Wong ${ }^{2}$ and Timothy D. Hau ${ }^{3}$ \\ ${ }^{I}$ Department of Geography, The University of Hong Kong, Pokfulam, Hong Kong \\ ${ }^{2}$ Department of Civil Engineering, The University of Hong Kong, Pokfulam, Hong Kong \\ ${ }^{3}$ School of Economics and Finance, The University of Hong Kong, Pokfulam, Hong Kong
}

\begin{abstract}
Should researchers collect choice or rank data in stated preference (SP) surveys? Answer to this question can have significant implications on survey costs and modeling outputs available for policy analysis. In particular, the exploded rank multinomial logit model (MNL) is compared with the ordinary choice-based MNL model. Using the empirical SP rank data collected among the public light bus operators in Hong Kong, the selected modeling approaches are compared in terms of model assumptions, model fit, modeling outputs and policy implications. Besides, the reliability of the exploded rank data is tested. The mixed results suggest that extra care must be exercised in the design of SP ranking tasks.
\end{abstract}

Keywords: Stated preference surveys, discrete choice, rank data, rank data reliability, MNL model.

\section{INTRODUCTION}

A review of the literature shows that the collection of discrete choice stated preference (SP) data was more common in transport surveys [1-4]. In contrast, rank data were collected less often. This was mainly attributable to modeling rather than survey difficulties [5-6]. To what extent are the less popular modeling techniques for rank data different from the discrete choice models? This paper attempts to compare selected rank- and choice-based modeling approaches in terms of model assumptions, model fit, modeling outputs and policy implications. The analysis is illustrated with the empirical data collected from a SP survey conducted in Hong Kong on the introduction of alternative fuel vehicles (AFVs) in the public light bus (PBL) industry. The results highlight the potential values and pitfalls of collecting rank data both from the survey and modeling perspectives.

\section{THEORETICAL FRAMEWORK}

A starting point for analyzing the multinomial logit (MNL) model is its theoretical foundation in utility maximization so that the probability of an individual $n$ choosing an element $i$ in a choice set $C_{n}$ consisting of $J_{n}$ choices is as follows:

$P_{n}(i)=\operatorname{Pr}\left(U_{i n} \geq U_{j n}, \forall j \in \mathrm{C}_{n}\right)$

where $\mathrm{P}_{n}(i)$ is the probability for $n$ to choose $i, \mathrm{U}_{i n}$ is the utility individual $n$ derived from element $i, j$ is an alternative within $\mathrm{C}_{n}$. In contrast to the individual-based conjoint analysis, the MNL model explicitly considers a stochastic disturbance term and results in a random utility model. The utility of each element consists of an observed (deterministic) component denoted by $V$ and a random (disturbance) component denoted by $\varepsilon$. Hence, equation (1) becomes:

*Address correspondence to this author at the Department of Geography, The University of Hong Kong, Pokfulam, Hong Kong;

Tel: (852)2859-7024; Fax: (852)2559-8994; E-mail: bpyloo@hkucc.hku.hk
$P_{n}(i)=\operatorname{Pr}\left(V_{i n}+\varepsilon_{i n} \geq V_{j n}+\varepsilon_{j n}, \forall j \in C_{n}, j \neq i\right)$

Moreover,

$U_{i n}=V_{i n}+\varepsilon_{i n}=\sum_{k=1}^{K} \varpi_{k n} x_{i k}+\varepsilon_{i n}$

where $\varpi_{k n}$ is the relative importance of attribute $k$ to individual $n, x_{i k}$ is the levels/values of attribute $k$ for alternative $i$. For each attribute, $\varpi_{k n}$ is assumed to be linear-in-parameter. In empirical studies, MNL models typically rely on the pooling of individual choice observations. With such pooling, the MNL models assume preference homogeneity among individuals so that they have identical relative attribute saliencies (Ortuzar and Willumsen 2001) [3]. When compared with conjoint analysis, the values of $\varpi_{k n}$ vary across alternatives but are the same for all individuals (fixed coefficients model). In relation, a limitation of the MNL model is that it is not possible to estimate the attribute weights at an individual level unless very large ranking tasks with 15 to 30 profiles are used to yield sufficient observations [7]. From the survey point of view, this would easily lead to respondent fatigue and substantially lower response rate and reliability. Hence, the values of $\varpi_{k n}$ are typically estimated for the attributes alone.

Maximum likelihood estimation methods are often used to estimate the attribute weights or coefficients for the choice model. It assumes that $\varepsilon$ is independently and identically distributed (IID) in accordance with the Gumbel distribution. In a simulated market share analysis, the explicit inclusion of $\varepsilon_{\text {in }}$ allows two apparent "irrationalities" to be explained [3]. Firstly, two individuals with the same socio-economic attributes facing the same choice set may make different choices. Secondly, individuals do not necessarily choose the alterna- 
tives with the highest expected utility. Moreover, the MNL model assumes that the ratio of the choice probability for the individual is unaffected by the systematic utilities of all other alternatives (independence from irrelevant alternatives, IIA, property). In this way, the probability that the individual will choose $i$ can be estimated like this:

$$
P_{i n}=\frac{e^{V_{i n}}}{\sum_{j \in J_{n}} e^{V_{j n}}}
$$

Developed within the context of a parametric distribution theory, the MNL coefficients (weight attributes) are fully metric in nature rather than simply relative attribute weights. This property allows statistical tests (such as significance of attribute weights) and estimations of choice probabilities with marginal changes in attribute levels to be made.

A discrete choice model, however, only accounts for the most preferred element $i$ among $J_{n}$ alternatives. Any additional information about individual $n$ 's ordinal rank preference data among the remaining $j$ alternatives, where $j \neq i$, is lost. To specify a rank probability model, one may begin with Luce's Choice Axiom that the random choice process of individual $n$ follows the top-to-bottom behavioral model with the same decision-making protocol. In other words, the rank orderings reflect a sequence of independent choice decisions. At the outset, the most preferable alternative is selected as rank number 1 . Then, the rest of the alternatives are considered as an independent and smaller choice set, the most preferable element is chosen and ranked number 2 . Subsequent rank orderings are determined in this way until $J_{n}-1$ choice sets are all independently considered.

When this top-to-bottom behavioral model is adopted, the decomposition of a rank probability follows the Luce and Suppes Ranking Choice Theorem [8] and is given by:

$P\left(1,2, \ldots, J_{n}\right)=P\left(1 \mid\left\{1,2, \ldots, J_{n}\right\}\right) * P\left(2 \mid\left\{2,3, \ldots, J_{n}\right\}\right) \ldots P\left(J_{n}-1 \mid\left\{J_{n}-1, J_{n}\right\}\right)$

$=\prod_{j=1}^{J_{n}-1} P\left(j \mid\left\{j, j=1, \ldots, J_{n}\right\}\right)$

where $P\left(1,2, \ldots, J_{n}\right)$ is the probability of having a rank order of alternative 1 as number 1 , alternative 2 as rank number 2 , and so on, and $P\left(j \mid\left\{j, j+1, \ldots J_{n}\right\}\right)$ is the probability of choosing alternative $j$ from the set of alternatives $\left\{j, j+1, \ldots J_{n}\right\}$. The sum of $J_{n}$-1 $\log$-likelihoods of choices, therefore, gives the log-likelihood of this event [9]:

$\ln P\left(1,2, \ldots, J_{n}\right)=\sum_{j=1}^{J_{n}-1} \ln P\left(j \mid\left\{j+1, \ldots, J_{n}\right\}\right)$

Moreover, the utility function of alternative $j$ for individual $n$ follows the random utility model (and, hence, the MNL model structure):

$U_{j n}=\hat{\beta} x_{j n}+\varepsilon_{j n}, j=1, \ldots, J_{n}$

where $x_{j n}$ is a vector of attributes of alternative $j$ for individual $n$ and $\hat{\beta}$ is the estimated vector of parameter estimates.

For lower rank observations, the probability that alternative $j$ is preferred to $j+1, \ldots, J_{n}$ is given by:
$P\left(j \mid\left\{j, j+1, \ldots J_{n}\right\}\right)=\frac{e^{\left[\theta\left(\hat{\beta}_{x_{j n}}\right)\right]}}{\sum_{i=j}^{J_{n}} e^{\left[\theta\left(\hat{\beta}_{x_{i n}}\right)\right]}}$

where $\theta$ is a scale parameter. Statistically, $\theta$ is inversely proportional to the standard deviation of the disturbance terms. In practice, it is usually normalized to be equal one.

The underlying assumption is that $\varepsilon_{j n}$ is IID in accordance with the same distribution form.

When compared to the discrete choice probability model, therefore, it is important to note that the rank choice probability model has two additional assumptions [10]. The first one is the top-to-bottom behavioral model with the same decision-making protocol (trade-offs among the underlying attributes) for ranking all alternatives. When this assumption is violated, an individual chooses by other principles, like the bottom-to-top procedure of successively eliminating inferior alternatives or the simple lexicographical decision rule of ranking on the basis of the most important attribute first and so on. The second additional assumption is that the disturbance terms of the first and all other subsequent ranks do not vary. In other words, the lower ranks should not be having greater error variance than higher ranks. When either of the above assumptions is violated, the reliability of the exploded rank data is in doubt.

While it is understood that the use of the rank MNL model (available, for example, in NLOGIT 4.0) does not require the tedious rank explosion of choice sets, it typically ignores the decision-making protocol of the respondents. More advanced rank MNL models allows alternative estimates to be generated by allowing a form of heteroscedasticity in the MNL specification so that higher ranked choices can be more precisely ranked than bottom ones [11]. Nonetheless, the rank MNL model yields no statistics to test whether the top-to-bottom behavioral model holds and whether the disturbance terms of different ranks are equal. Nonetheless, answers to these questions are valuable to researchers deciding whether to collect rank or discrete choice data. Moreover, if rank data are collected, how many ranks can be used? How reliable are SP data at the lower ranks? Using the exploded rank MNL model, it is hoped that the relative advantages and disadvantages of collecting rank or discrete choice data can be compared more systematically by using the same set of SP data.

\section{THE EMPIRICAL SURVEY}

In mid-2002, an industry-wide SP survey was conducted in Hong Kong to gather the views of PLB operators on the introduction of AFVs in their industry [12]. One of the aims of the SP survey was to inform policy makers on the formulation of efficient and cost-effective measures to facilitate a replacement of the diesel PLB fleet by the liquefied petroleum gas (LPG) PLB fleet. During this survey, about 2,500 questionnaires were distributed among the PLB operators. Four SP games, involving two different designs, were included in the questionnaire. In each SP task, respondents were requested to provide a complete rank ordering of all choice set alternatives rather than to make a single choice 
only. The first SP design simulated a vehicle-replacement decision of the PLB operators by providing the respondents with 4 vehicle designs. Each vehicle design was characterized by 8 underlying attributes of fuel type, fuel price, vehicle price, distance to the nearest refueling station, vehicle range, vehicle life, seats and horse power. The second SP design asked the respondents to rank 3 different government support packages. The government support packages were differentiated by 7 underlying attributes of vehicle subsidy, low interest-rate loan, fuel subsidy, distance to the nearest refueling station, vehicle range, horse power and seats. After data editing, a total of 483 completed questionnaires were collected. Due to response omissions, 903 and 815 valid responses were received for the SP designs of vehicle replacement and government support respectively.

\section{INSIGHTS FROM MNL ANALYSIS}

In this paper, the discrete choice and exploded rank MNL models are used to analyze the same set of SP rank data. Details of the survey logistics and the discrete choice model have been reported in Loo, Wong and Hau (2006) [12]. Following the discussion in Section 2, the preference rank data are first exploded $[8,10]$. For the vehicle-replacement decision, Table 1 shows the summary MNL results of the exploded rank data. In relation, Table 2 shows the summary MNL results of the discrete choice model (first rank used as the discrete choice). The summary results of the exploded rank and choice MNL models for government support are shown in Tables $\mathbf{3}$ and $\mathbf{4}$ respectively.

The most obvious difference is that the levels of statistical significance of MNL coefficients are systematically higher for the exploded rank model. Furthermore, the standard errors for all parameter estimates in both SP games have reduced rather substantially. These desirable properties are considered one of the most important advantages of using exploded rank data from the modeling perspective [7, 10]. The use of more independent choice sets from each respondent leads to reduced sampling variance. When the sampling variance is lower, the accuracy of parameter estimates is higher, ceteris paribus.

Table 1. Results from the Exploded Rank MNL Model for Vehicle Replacement

\begin{tabular}{|l|c|c|c|}
\hline & Coefficient & Standard Error & $\boldsymbol{t}$-Stat. \\
\hline \hline Fuel type & $-3.35^{*}$ & 0.38 & -8.82 \\
\hline Fuel price & $-0.10^{*}$ & 0.08 & -12.52 \\
\hline Vehicle price & $-0.11-04^{*}$ & $3.88 \mathrm{E}-05$ & -2.92 \\
\hline Distance & $-0.24^{*}$ & 0.03 & -7.42 \\
\hline Range & $0.00^{*}$ & 0.00 & 5.83 \\
\hline Vehicle life & $0.27 *$ & 0.04 & 6.83 \\
\hline Seats & $0.54 *$ & 0.04 & 13.10 \\
\hline Horse power & $0.02 *$ & 0.00 & 5.40 \\
\hline
\end{tabular}

To gain an idea about the magnitude of change associated with changes in an attribute, the MNL coefficients of the
Table 2. Results from the Discrete Choice MNL Model for Vehicle Replacement

\begin{tabular}{|l|c|c|c|}
\hline & Coefficient & Standard Error & $\boldsymbol{t}$-Stat. \\
\hline \hline Fuel type & $-5.11^{*}$ & 0.69 & -7.42 \\
\hline Fuel price & $-1.06^{*}$ & 0.14 & -7.39 \\
\hline Vehicle price & 0.00 & 0.00 & -0.19 \\
\hline Distance & $-0.21 *$ & 0.05 & -4.18 \\
\hline Range & $0.00^{*}$ & 0.00 & 2.83 \\
\hline Vehicle life & $0.28^{*}$ & 0.07 & 3.97 \\
\hline Seats & $0.70^{*}$ & 0.07 & 9.36 \\
\hline Horse power & $0.02^{*}$ & 0.01 & 3.32 \\
\hline
\end{tabular}

Table 3. Results from the Exploded Rank MNL Model for Government Support

\begin{tabular}{|l|c|c|c|}
\hline & Coefficient & Standard Error & $\boldsymbol{t}$-Stat. \\
\hline \hline Vehicle subsidy & $3.22 \mathrm{E}-05^{*}$ & $5.46 \mathrm{E}-06$ & 5.90 \\
\hline Loan & $-0.28^{*}$ & 0.05 & -5.60 \\
\hline Fuel subsidy & $0.54^{*}$ & 0.14 & 3.95 \\
\hline Distance & $-0.81^{*}$ & 0.12 & -6.52 \\
\hline Range & $0.01^{*}$ & 0.00 & 8.12 \\
\hline Horse power & $0.04^{*}$ & 0.01 & 5.46 \\
\hline Seats & $0.43^{*}$ & 0.05 & 9.09 \\
\hline
\end{tabular}

Table 4. Results from the Discrete Choice MNL Model for Government Support

\begin{tabular}{|c|c|c|c|}
\hline & Coefficient & Standard Error & $\boldsymbol{t}$-Stat. \\
\hline \hline Vehicle subsidy & $3.04 \mathrm{E}-05^{*}$ & $7.04 \mathrm{E}-06$ & 4.32 \\
\hline Loan & $-0.24^{*}$ & 0.07 & -3.62 \\
\hline Fuel subsidy & $0.64^{*}$ & 0.18 & 3.65 \\
\hline Distance & $-0.90^{*}$ & 0.16 & -5.55 \\
\hline Range & $0.01^{*}$ & 0.00 & 6.14 \\
\hline Horse power & $0.03^{*}$ & 0.01 & 3.25 \\
\hline Seats & $0.42^{*}$ & 0.06 & 7.41 \\
\hline
\end{tabular}

* Significant at the 0.05 level.

exploded rank and discrete choice models are compared. For the government support SP design, the MNL results of the exploded rank and choice models are similar with seats and range being the most statistically significant. Nonetheless, the MNL coefficients are of higher statistical significance with lower standard errors and higher $t$-statistics. For the vehicle SP game, the importance of vehicle price is much higher for the exploded rank than the choice model. In the latter, vehicle price is not significant at the 0.05 level. Over- 
all, the discrete choice model points to seats, fuel type and fuel price as the most significant variables in the vehiclereplacement decisions. In the exploded rank data model, though the number of seats remains the most significant, fuel price is having the second higher t-statistics very close to seats. However, how reliability are the additional rank data beyond rank 1 ? Would the data reliability differ for the two SP games?

\section{RANK DATA RELIABILITY}

Generally, the existing literature suggests that rank data at lower ranks may be "nosier", that is, having a large error variance. In this survey, each vehicle-replacement SP game has 4 alternatives. When the first rank is taken as the discrete choice, the MNL results are identical to the choice model and the depth of explosion $(E)$ is considered to be $1(E=1)$. Following [9], this choice set is called Data Set 1 . When $E=2$, the exploded choice set is Data Set 1 plus Data Set 2 . Data Set 2 is restricted to all but the first rank element and the choice refers to rank number 2 . As there are 4 alternatives in the vehicle-replacement SP game, the maximum depth of data explosion is $3(\operatorname{Max}(E)=3)$. Similarly, the 3 alternatives of government support packages mean that the maximum depth of data explosion is $2(\operatorname{Max}(E)=2)$.

In order to test the reliability of the exploded rank data, the equality of individual coefficients is first tested statistically. The null hypothesis $\left(\mathrm{H}_{0}\right)$ is that there is no significant difference between the coefficient estimates for different Data Sets. The asymptotically normal test statistic, $\lambda$, is used to test the equality of a particular MNL coefficient:

$\lambda=\frac{\beta_{k}^{1}-\beta_{k}^{2}}{\sqrt{\operatorname{Var}\left(\beta_{k}^{1}\right)+\operatorname{Var}\left(\beta_{k}^{2}\right)}}$

where $\beta_{k}$ is the coefficient of the $k$ th attribute, $\hat{\beta}_{k}^{1}$ and $\hat{\boldsymbol{\beta}}_{k}^{2}$ refer to the estimates of $\beta_{k}$ from Data Set 1 and 2 respectively, and $\operatorname{Var}\left(\hat{\beta}_{k}^{1}\right)$ and $\operatorname{Var}\left(\hat{\beta}_{k}^{2}\right)$ are the values of variance of $\hat{\boldsymbol{\beta}}_{k}^{1}$ and $\hat{\boldsymbol{\beta}}_{k}^{2}$ respectively [9]. A two-tail test is used and the level of statistical significance chosen is 0.05 . The critical Z-score $\left(Z_{\alpha / 2}\right)$ is $\pm 1.96 . \mathrm{H}_{0}$ is rejected when $\lambda$ falls outside this critical range. Rejection of $\mathrm{H}_{0}$ means that the parameter estimates vary substantially across different ranks and different decision protocols might have been used by the respondents. Under such circumstances, the exploded rank data do not conform to the Luce and Suppes Choice Theorem and the additional information from the exploded ranking data may not be reliable. The summary results of the equality of individual coefficients tests for the vehicle replacement and the government support SP games are shown in Tables 5 and $\mathbf{6}$ respectively.

For the vehicle-replacement SP design, three cases are tested: firstly, between the first (Data Set 1) and second ranks (Data Set 2); secondly, between the second and third ranks (Data Set 3); and lastly, between the first and third ranks. Table 5 shows that $\mathrm{H}_{0}$ are rejected in 9 out of the 21 tests conducted. In particular, $\mathrm{H}_{0}$ are rejected for all attrib-
Table 5. Summary Results of Equality of Individual Coefficients Tests for Vehicle Replacement

\begin{tabular}{|l|c|c|c|}
\hline \multirow{2}{*}{} & \multicolumn{3}{|c|}{$\lambda$} \\
\cline { 2 - 4 } & $\begin{array}{c}\text { Data Set 1 vs } \\
\text { Data Set 2 }\end{array}$ & $\begin{array}{c}\text { Data Set 1 vs } \\
\text { Data Set 2 }\end{array}$ & $\begin{array}{c}\text { Data Set 1 vs } \\
\text { Data Set 2 }\end{array}$ \\
\hline \hline Fuel type & -1.80 & -0.59 & $-2.27 \#$ \\
\hline Fuel price & 1.52 & -1.30 & 0.10 \\
\hline Vehicle price & $-12.26 \#$ & 1.83 & $-9.67 \#$ \\
\hline Distance & 0.58 & $-2.62 \#$ & $-2.34 \#$ \\
\hline Range & -1.43 & 1.30 & 0.11 \\
\hline Vehicle life & -0.66 & $2.50 \#$ & 1.91 \\
\hline Seats & 1.00 & 1.62 & $2.50 \#$ \\
\hline Horse power & -0.09 & $2.12 \#$ & $2.35 \#$ \\
\hline
\end{tabular}

Table 6. Summary Results of Equality of Individual Coefficients Tests for Government Support

\begin{tabular}{|c|c|}
\hline & $\boldsymbol{\lambda}$ (Data Set 1 vs Data Set 2) \\
\hline \hline Vehicle subsidy & -0.20 \\
\hline Loan & 0.52 \\
\hline Fuel subsidy & 0.48 \\
\hline Distance & -0.46 \\
\hline Range & -0.09 \\
\hline Horse power & -0.72 \\
\hline Seats & 0.23 \\
\hline Note: Critical Z-score is \pm 1.96. &
\end{tabular}

utes, except the fuel price, range and vehicle life, in the comparison of the MNL coefficients between Data Sets 1 and 3. Similarly, the tests of difference were conducted between the first and second ranks for the government support $\mathrm{SP}$ game. Table 6 shows that $\mathrm{H}_{0}$ cannot be rejected for all 7 tests at the 0.05 level. In other words, the MNL coefficients for each attribute do not vary significantly across different Data Sets for the government support SP games.

When all attributes of the MNL model are taken as a whole, the Watson and Westin pooling test is conducted. The null hypothesis $\left(\mathrm{H}_{0}\right)$ is that the coefficient vectors of different data sets are not statistically different at the 0.05 level. To conduct the Watson and Westin pooling tests, the loglikelihood ratios of the MNL models for different Data Sets and the likelihood ratio test statistic are calculated:

$$
L R=-2\left(L_{R}-L_{U}\right)
$$

where $L_{R}$ is the log-likelihood for the restricted model; and $L_{U}$ is the log-likelihood for the unrestricted model. The former is estimated by pooling the different data sets for the MNL analysis. To illustrate, $\mathrm{L}_{1+2}(\hat{\boldsymbol{\beta}})$ refers to the $\log$ likelihood for the pooled data model of Data Sets 1 and 2 
combined. The latter is given by the sum of the loglikelihoods of the MNL models estimated separately for the data sets, that is $\mathrm{L}_{1}(\hat{\boldsymbol{\beta}})+\mathrm{L}_{2}(\hat{\boldsymbol{\beta}})$, where $\mathrm{L}_{1}(\hat{\boldsymbol{\beta}})$ and $\mathrm{L}_{2}(\hat{\boldsymbol{\beta}})$ are the log-likelihood values for Data Sets 1 and 2 respectively. The test statistic follows the $\chi^{2}$ distribution. The degree of freedom $(d f)$ is $K_{U}-K_{R}$, where $K_{U}$ and $K_{R}$ are the number of estimated parameters in the unrestricted and restricted models respectively. The level of statistical significance chosen is 0.05 . From the $\chi^{2}$ distribution table, the Critical $\chi^{2}$ can be looked up. $\mathrm{H}_{0}$ is rejected when the test statistic is larger than Critical $\chi^{2}$. The overall summary results are shown in Table 7.

Table 7. Results of the Watson and Westin Pooling Tests

\begin{tabular}{|c|c|c|c|c|}
\hline & \multicolumn{4}{|c|}{ Vehicle-Replacement SP Design } \\
\cline { 2 - 5 } & LR & $\boldsymbol{d f}$ & Critical $\chi^{2}$ & $\boldsymbol{H}_{\boldsymbol{\theta}}$ \\
\hline \hline Data Sets 1 \& 2 & 205.3 & 8 & 15.51 & Reject \\
\hline Data Sets 2 \& 3 & 22.56 & 8 & 15.51 & Reject \\
\hline Data Sets 1,2\&3 & 366.0 & 16 & 26.3 & Reject \\
\hline & \multicolumn{4}{|c|}{ Government Support SP Design } \\
\hline Data Sets 1 \& 2 & 5.81 & 7 & 14.07 & Do not reject \\
\hline
\end{tabular}

For the Watson and Westin pooling tests, $\mathrm{H}_{0}$ are rejected in all three cases for the vehicle-replacement SP game. In other words, the MNL coefficient vectors for the different data sets in this SP game are statistically different at the 0.05 level. This implies that the different data sets are likely to have come from different underlying choice process, with different error term structures. Yet, it is not possible to tell whether the top-to-bottom behavioral model or the same error distribution assumption has been violated [9]. For the government support SP game, $\mathrm{H}_{0}$ is not rejected at the 0.05 level. In other words, the parameter vectors estimated from Data Sets 1 and 2 are not significantly different; and that the full information from the SP ranking exercise can be fruitfully analyzed by the exploded MNL model, without violating the top-to-bottom behavior and same error distribution assumptions [10].

The different Watson and Westin pooling test results of the two SP games highlight the need to be judicious about the collection and analysis of preference rank data. Moreover, the reliability of the rank data may also be affected by the number of alternatives presented to the respondents in the ranking exercise. Previous literature suggests that an explosion depth beyond 3 needs to be carefully considered [10]. The present study shows that further studies about the optimal explosion depth may be required. For instance, the explosion depth of 2 was suitable for the government SP game but the same depth was not reliable for the vehiclereplacement SP game. Two major differences between the SP games are that 1) the number of attributes of the vehicle replacement game (eight) was higher than the government support (seven); and 2) the number of alternatives presented in the vehicle replacement choice basket (four) was higher than the government support choice basket (three). The presentation of a smaller choice set for the ranking exercise might have induced the respondents to carefully consider all different options in the choice baskets. Taking into consideration that each alternative is characterized by as many as 78 attributes, the presentation of a slightly larger choice set (4 instead of 3) could have reduced the incentives for respondents to consider each option and each rank seriously. While the overall satisfactory response rate of the SP survey shows that SP ranking tasks are not too onerous and can be suitable for respondents of different intellectual ability, the design of the SP games is crucial in affecting the quality of the SP rank data collected. Generally, data at the same rank can be "nosier" for choice baskets with a larger number of alternatives.

\section{CONCLUDING REMARKS}

Asking respondents to rank their preferences has many potential advantages. At the data collection stage, "obtaining additional choice sets from independent decision makers is generally more expensive than asking a smaller set of decision makers to supply the complete preference rank ordering of all elements in their choice sets" (Chapman and Staelin 1982, p. 291) [10]. Surveys are expensive in terms of human resource, money and time. This is particularly the case for SP surveys because the inclusion of SP tasks often means lower response rates and more expensive survey methods (for example, face-to-face surveys). At the modeling stage, rank data can be analyzed by different methods. This paper systematically compares the theoretical foundations and empirical results of two selected MNL modeling approaches. In particular, the use of full rank data increases the precision of parameter estimates through lowering standard errors and raising levels of statistical significance. Nonetheless, the reliability of the rank data rests on two assumptions. First, the respondents follow the top-to-bottom behavioral model and use the same decision-making protocol in ranking all alternatives. Second, the error term distribution is the same across different ranks. In the SP survey conducted in Hong Kong, it was found that these two additional assumptions may not hold. Moreover, the different results of the two SP games highlight the need for researchers to be careful about the collection and analysis of rank data in their specific circumstances. So far, there is no conclusive evidence about the reliability of rank data in SP surveys. On the one hand, researchers should not be prejudiced against the collection and analysis of preference rank data. On the other hand, researchers should be cautious about the use of the rank data without testing for their reliability.

\section{REFERENCES}

[1] M. Ben-Akiva, Discrete Choice Analysis: Theory and Applications to Travel Demand. Cambridge: MIT Press, 1985.

[2] J.J. Louviere, D.A. Hensher and J.D. Swait, Stated Choice Methods: Analysis and Application. Cambridge: Cambridge University Press, 2000.

[3] J. de D. Ortuzar and L.G. Willumsen, Modelling Transport. Chichester: John Wiley \& Sons, 2001.

[4] B.P.Y. Loo, "Role of stated preference methods in planning for sustainable urban transportation: State of practice and future prospects", J. Urban. Plann. Dev., vol.128, No.4, pp. 210-24, December 2002 .

[5] S.D. Beggs and N.S. Cardell, "Choice of smallest car by multivehicle households and the demand for electric vehicles", Transport. Res. A., vol. 14, pp. 389-404, 1980.

[6] P.E. Green and V. Srinivasan, "Conjoint analysis in marketing: New developments with implications for research and practice", $J$. Market., vol. 54, No. 4, pp. 3-19, October 1990.

[7] S. Beggs, S. Cardell and J. Hausman, "Assessing the potential demand for electric cars”, J. Econom., vol. 16, pp. 1-19, 1981. 
[8] R.D. Luce and P. Suppes, "Preference, utility, and subjective probability", in Handbook of Mathematical Psychology, R.D. Luce, R.R. Bush and E. Galanter, Eds. New York: John Wiley and Sons, 1965, pp. 249-410.

[9] M. Ben-Akiva, T. Morikawa and F. Shiroishi, "Analysis of the reliability of preference ranking data", J. Bus. Res., vol. 24, pp. 149-164, November 1992.
[10] R.G. Chapman and R. Staelin, "Exploiting rank-ordered choice set data within the stochastic utility model", J. Mark. Res., vol. 19, No. 3, pp. 288-301, August 1982.

[11] J.A. Hausman and P.A. Ruud, "Specifying and testing econometric models for rank-ordered data", J. Econom., vol. 34, pp. 83-104. 1987.

[12] B.P.Y. Loo, S.C. Wong and T.D. Hau, "Introducing alternative fuel vehicles in Hong Kong: Views from the public light bus industry", Transport, vol. 33 (6), pp. 605-619, 2006.

Received: May 21, 2008

(c) Loo et al.; Licensee Bentham Open.

This is an open access article licensed under the terms of the Creative Commons Attribution Non-Commercial License (http://creativecommons.org/licenses/by$\mathrm{nc} / 3.0 /$ /) which permits unrestricted, non-commercial use, distribution and reproduction in any medium, provided the work is properly cited. 